\title{
Time domain design of fractional differintegrators using least-squares
}

\author{
Ramiro S. Barbosa, J.A. Tenreiro Machado, Manuel F. Silva
}

\begin{abstract}
In this paper we propose the use of the least-squares based methods for obtaining digital rational approximations (IIR filters) to fractional-order integrators and differentiators of type $s^{\mathrm{a}}$, a2R. Adoption of the Pade', Prony and Shanks techniques is suggested. These techniques are usually applied in the signal modeling of deterministic signals. These methods yield suboptimal solutions to the problem which only requires finding the solution of a set of linear equations. The results reveal that the least-squares approach gives similar or superior approximations in comparison with other widely used methods. Their effectiveness is illustrated, both in the time and frequency domains, as well in the fractional differintegration of some standard time domain functions.
\end{abstract}

Keywords

Fractional-order control; IIR filter; Rational approximations; Digital differentiators; Digital integrators; T-integrator; Discretization

\section{Introduction}

The area of fractional calculus (FC) emerged, three centuries ago, at the same time as the classical differential calculus and deals with derivatives and integrals to an arbitrary order: real, rational, irrational or even complex order [1-3]. However, its inherent complexity postponed the application of the associated concepts. Nowadays, the FC theory is applied in almost all the areas of science and engineering, being recognized its ability to yield a superior modeling and control in many dynamical systems [1,3-6].

In what concerns the area of control systems the application of the FC concepts is still scarce and only in the second-half of the last century appeared the first applications. Oustaloup [4] introduced the fractional-order algorithms and demonstrated the superior performance of the CRONE controller (French abbreviation of "Commande Robuste d'Ordre Non Entier'") over the standard PID controller. More recently, Podlubny [3,7] proposed a generalization of the PID scheme, namely the $\mathrm{PI}^{1} \mathrm{D}^{\mathrm{m}}$ controller, involving an integrator of order 1 and differentiator of order $\mathrm{m}$ (the orders 1 and $\mathrm{m}$ may assume real noninteger values). $\mathrm{He}$ also 
demonstrated the superior response of this type of controller, in comparison with the classical PID, when used for the control of fractional-order systems. The transfer function of the $\mathrm{PI}^{\mathrm{l}} \mathrm{D}^{\mathrm{m}}$ is given by $\left.K / 1 \mathbf{p} ð 1=T_{i} s^{-1} \mathbf{p} T_{d} s^{\mathrm{m}}\right]$, where 1 and $\mathrm{m}$ are positive real numbers; $K$ is the proportional gain, $T_{i}$ the integral time constant and $T_{d}$ the derivative time constant. Clearly, taking $ð 1 ; \mathrm{mP} 1 / 4 \mathrm{f} ð 1 ; 1 \mathrm{p} ; \succsim 1 ; 0 \mathrm{p} ; 00 ; 1 \mathrm{p}$; $0 ; 0 \mathrm{Pg}$ we obtain the classical PID; PI; PD; P g controllers, respectively. All these classical types of PID algorithms are particular cases of the fractional $\mathrm{PI}^{1} \mathrm{D}^{\mathrm{m}}$ controller. However, the $\mathrm{PI}^{1} \mathrm{D}^{\mathrm{m}}$ controller is more flexible and gives the possibility of adjusting more carefully the dynamical properties of a fractional-order control (FOC) system.

The fundamental element of the FOC strategies is

the fractional-order differentiator and/or integrator (hereafter referred to as differintegrator), $2 \mathrm{R} \mathrm{p}$. Hence, the crucial step in digital implementation of an FOC is the discretization of the fractional differintegrator $s^{\text {a }}$. In this study, the approach for obtaining discrete transfer functions approximations to fractional differintegrators adopts the tions to fractional differintegrators adopts the techniques of Pade', Prony and Shanks. These techniques are usually applied in the signal modeling of deterministic signals. The whole process can be summarized in the following three steps:

(1) Discretize the fractional-order operator $s^{\mathrm{a}}$ using a suitable generating function $s^{\mathrm{a}} 1 / 4 H^{\mathrm{a}} \Varangle^{-1} ;$ p

(2) Obtain the impulse response sequence $h^{\text {a }} \not k$, of the fractional discrete equivalent, by performing a power series expansion (PSE) (or Taylor series) over $H^{\mathrm{a} d} z^{-1} \mathrm{p}$;

(3) Apply the signal modeling techniques (Pade', Prony or Shanks) to the impulse response sequence $h^{\text {ax }} k$ pin order to get the desired IIRtype approximation.

The least-squares strategy just described provides rational transfer functions of the $z$ variable that give good approximations, both in the time and frequency domains, to continuous fractional-order operators. Therefore, they represent an alternative choice to other proposed methods, namely the widely used continued fraction expansion (CFE) method.

Bearing these ideas in mind, the paper is organized as follows. Section 2 reviews the fundamentals of FC. Section 3 presents an unified discretization scheme for fractional-order integrators and differentiators, while Section 4 derives its impulse response. Section 5 develops the signal modeling techniques of Pade', Prony and Shanks for the design of IIR approximations to continuous fractional-order operators. Section 6 presents some illustrative examples showing the effectiveness of the proposed techniques. Finally, Section 7 draws the main conclusions.

\section{Essentials of fractional calculus}

In the literature we find several different definitions for differentiation and integration to an arbitrary order [1-3]. One usually defines the generalized operator by the notation ${ }_{a} D_{t}^{\mathrm{a}}$, where $a$ and $t$ are the limits and a $\mathrm{\gamma a}^{2} \mathrm{RP}$ the order of the operation. The two most well-known definitions are the Riemann-Liouville and the Gru"nwald-Letnikov definitions which for a wide class of functions are equivalent. The Riemann-Liouville definition is given by (a 40p:

$$
\begin{aligned}
{ }_{a} D_{t}^{\alpha} f(t) & =\frac{1}{\Gamma(n-\alpha)} \frac{d^{n}}{\mathrm{~d} t^{n}} \int_{a}^{t} \frac{f(\tau)}{(t-\tau)^{\alpha+1-n}} \mathrm{~d} \tau, \\
n-1 & <\alpha<n,
\end{aligned}
$$

where $G \&$ represents the Gamma function of $x$. From a control and signal processing perspective, the definition of fractional differintegration given by the Gru"nwald-Letnikov approach seems to be the most useful and intuitive, particularly for a discretetime implementation $[3,8,9]$. It is defined by the following expression ða $2 \mathrm{RP}$ :

${ }_{a} D_{l}^{\alpha} f(t)=\lim _{h \rightarrow 0} \frac{1}{h^{\alpha}} \sum_{k=0}^{[t-\alpha / h]}(-1)^{\alpha}\left(\begin{array}{l}\alpha \\ k\end{array}\right) f(t-k h)$,

$\left(\begin{array}{l}\alpha \\ k\end{array}\right)=\frac{\Gamma(\alpha+1)}{\Gamma(k+1) \Gamma(\alpha-k+1)}$,

where $f$ tois the applied function, $h$ is the time increment and $[x]$ means the integer part of $x$. Oldham and Spanier [1] called operator (2) a differintegral since it unifies in a single operator the notions of derivative and integral. Furthermore, the Gru"nwald-Letnikov definition poses the fewest restrictions on the functions upon which it is applied and can be converted easily into numerical schemes. An important property revealed by Eqs. (1) and (2) is that while integer-order operators imply a finite series, the fractional-order counterparts are defined by an infinite series. This means that integer operators are local operators in opposition with the 
fractional operators that have, implicitly, a "memory" of all past events.

For the analysis and synthesis of automatic control systems we often adopt the Laplace transform $(L)$ based methods. In the Laplace domain, the fractional differintegration of order $\alpha \in \Re,{ }_{a} D_{l}^{z} f(t)$, of $f(t)$ (being $f(t)$ a causal function of $t, f(t)=0$ for $t<a>0$ ), under null initial conditions, is given by the simple expression:

$$
\left.L_{{ }_{a}} D_{l}^{\alpha} f(t)\right\}=s^{\alpha} F(s),
$$

where $F(s)=L\{f(t)\}$. Note that expression (3) is a direct generalization of the classical integer-order scheme with the multiplication of the signal transform $F(s)$ by the Laplace $s$-variable raised to a fractional value $\alpha$. The Bode diagrams of amplitude and phase of the fundamental fractional-order operator $s^{x}$ (3) are represented by straight lines of $20 \alpha \mathrm{dB} / \mathrm{dec}$ and $\alpha \pi / 2 \operatorname{rad}(\alpha \in \Re)$ in all frequency domain, respectively. These facts reveal that frequency-based analysis methods may be easily adapted to the fractional-order case.

3. Unified discretization scheme for fractional-order differentiators and integrators

The usual approach for obtaining discrete equivalents of continuous fractional-order operators of type $s^{\alpha}(\alpha \in \Re)$ adopts a generating function $s=\omega\left(z^{-1}\right)[10,11]$. By other words, given a continuous transfer function (filter), $G(s)$, a discrete equivalent, $G(z)$, can be found through the substitution:

$G(z)=\left.G(s)\right|_{s^{s}=H^{z}(z)}$,

where $H^{\alpha}(z)$ denotes the fractional discrete equivalent of order $\alpha$ of fractional-order operator $s^{z}$, expressed as a function of the complex variable $z$ or of the shift operator $z^{-1}$. In these $s \rightarrow z$ conversion schemes (also called analog to digital open-loop design methods) the most often used are the Euler (first backward difference rule), the Tustin (trapezoid rule) and the more recently introduced AlAlaoui operator, which is a weighted interpolation of the Euler integration rule (3) and the Tustin in tegration rule $\left(\frac{1}{4}\right)[12,13]$.

It can be shown that the mentioned numerical integration formulas are special cases of the socalled T-integrator introduced by Smith and described in his book [14]. In fact, Smith defined a new type of integration formula, which has a close relation to the mean value theorem, given by the two-parameter tunable transfer function:

$$
\frac{1}{s}=\frac{\lambda T\left[\gamma+(1-\gamma) z^{-1}\right]}{1-z^{-1}},
$$

where $\lambda$ and $\gamma$ are denoted the gain and phase tuning parameters, respectively. An important aspect of the T-integrator is that for $\lambda=1$ and varying $\gamma$ from 0 to 2 in ratios of integers results in most of the useful classical first order integrators. For example, when $\lambda=1$ and $\gamma=\left\{\frac{1}{2}, \frac{7}{8}, 1\right\}$, the T-integrator (5) becomes the Tustin, the Al-Alaoui and the Euler (backward difference) integration rules, respectively. More specifically, the T-integrator can take on a double infinity of values ( $\lambda$ and $\gamma$ ) in-between the classical integrators. In this sense the integrator can be tuned precisely to any problem being solved, system being controlled or system being simulated [14]. Thus, the designation of Tunable-type integrators (T-integrators).

We can obtain a family of new fractional-order differentiators from the digital $\mathrm{T}$-integrator (5). The direct inversion of (5), raised to the power a, will give the following generating function for discretization:

$$
H^{\alpha}(z)=\left(\frac{1}{\lambda T} \frac{1-z^{-1}}{\gamma+(1-\gamma) z^{-1}}\right)^{\alpha} .
$$

Table 1 lists some of the fractional discretization schemes that results from the unified generating function (6) for different values of parameter $g$ (with a fixed value of 11/41). Clearly, many of the widely varied classical numerical integration formulas, each of which is considered in some way to be different from the others, are actually the same integrator, differing only in the amount of phase shift g. For a more detailed description about the features of this integrator see [14].

\section{Table 1}

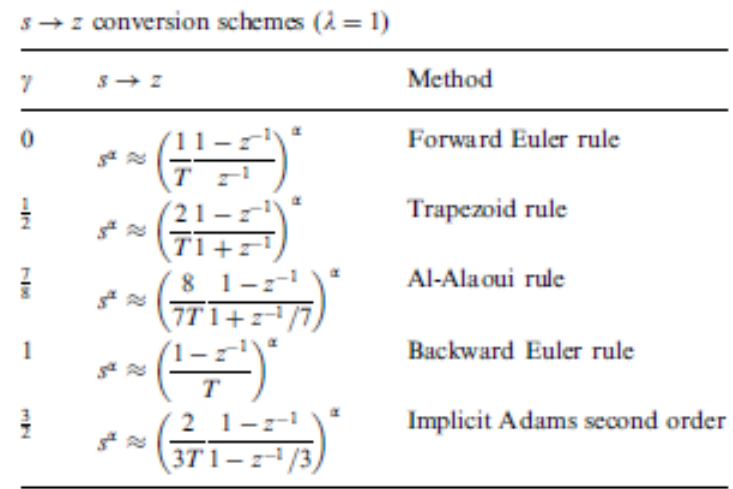


As can be seen in Table 1, the fractional-order conversion schemes lead to nonrational $z$-formulae. In order to get rational expressions we have two alternatives. One way is to perform a PSE (Taylor series) over them and the final approximation corresponds to a truncated $z$-polynomial function (FIR filter) [8-10,15]. For example, using the backward Euler rule, $H\left(z^{-1}\right)=\left(1-z^{-1}\right) / T$, and performing a PSE of $\left(\left(1-z^{-1}\right) / T\right)^{\alpha}$ gives the discretization formula corresponding to the Grünwald-Letnikov definition (2), yielding:

$$
\begin{aligned}
D^{\alpha}(z) & =\frac{Y(z)}{X(z)}=\left(\frac{1}{T}\right)^{\alpha} \operatorname{PSE}\left\{\left(1-z^{-1}\right)^{\alpha}\right\}_{N} \\
& =\left(\frac{1}{T}\right)^{\alpha} P_{N}\left(z^{-1}\right) \\
& =\left(\frac{1}{T}\right)^{\alpha}\left(c_{0}^{(\alpha)}+c_{1}^{(\alpha)} z^{-1}+\cdots+c_{N}^{(\alpha)} z^{-N}\right),
\end{aligned}
$$

where $P$ is a polynomial of degree $N$ and $c_{k}^{(\alpha)},(k=$ $0,1, \ldots)$ are binomial coefficients which may be calculated recursively as:

$$
c_{0}^{(\alpha)}=1, \quad c_{k}^{(\alpha)}=\left(1-\frac{1+\alpha}{k}\right) c_{k-1}^{(\alpha)}, k=1,2, \ldots .
$$

Another possible way is to obtain a discrete transfer function in the form of rational function (i.e., as the ratio of two polynomials) (IIR filter). This can be accomplished by application of the well-known CFE method [10,11,16-18]. By doing so, over the tunable generating function (6), it results in the discrete transfer function, approximating continuous fractional-order operators, expressed as:

$$
\begin{aligned}
D^{\alpha}(z) & =\frac{Y(z)}{X(z)}=\left(\frac{1}{\lambda T}\right)^{\alpha} \operatorname{CFE}\left\{\left(\frac{1-z^{-1}}{\gamma+(1-\gamma) z^{-1}}\right)^{\alpha}\right\}_{m, n} \\
& =\left(\frac{1}{\lambda T}\right)^{\alpha} \frac{P_{m}\left(z^{-1}\right)}{Q_{n}\left(z^{-1}\right)} \\
& =\left(\frac{1}{\lambda T}\right)^{\alpha} \frac{p_{0}+p_{1} z^{-1}+\cdots+p_{m} z^{-m}}{q_{0}+q_{1} z^{-1}+\cdots+q_{n} z^{-n}}
\end{aligned}
$$

where $P$ and $Q$ are polynomials of degree $m$ and $n$, respectively. It is well known that rational approximations frequently converge faster than polynoconvergence in the complex domain. Therefore, in this study we only develop $z$-variable rational transfer functions approximations of the continuous fractional-order operators. Moreover, the proposed algorithms adopt the time domain, which make them suited for $z$-transform analysis and discretetime implementation.

The CFE method leads to an approximation that produces a Taylor series coefficients identical to those obtained through the fractional-order discrete equivalent up to the order of approximation, that is, $D^{z}(z)-H(z)=\mathrm{O}\left(z^{m+n+1}\right)$, where $H(z)$ is the approximation of order $m+n+1[11,19]$. The new techniques relax this constraint by considering the least-squares determination of the model parameters over a wider range of the Taylor series coefficients (or of the impulse response length).

4. Impulse response of digital fractional-order differentiators and integrators

This section derives the impulse response sequence $h^{\alpha}(k)$ corresponding to the tunable generating function $H^{\alpha}\left(z^{-1}\right)$ (6). It is assumed that $h^{\alpha}(k)=0$ for $k<0$, that is, a causal system.

One puts the discretization formula $H^{\alpha}\left(z^{-1}\right)$ in the form:

$$
\begin{aligned}
H^{\alpha}\left(z^{-1}\right) & =\left(\frac{1}{\lambda T} \frac{1-z^{-1}}{\gamma+(1-\gamma) z^{-1}}\right)^{\alpha} \\
& =\left(\frac{1}{\lambda T}\right)^{\alpha}\left(1-z^{-1}\right)^{\alpha}\left(\gamma+(1-\gamma) z^{-1}\right)^{-\alpha} .
\end{aligned}
$$

By taking the PSE of the functions $\left(1-z^{-1}\right)^{\alpha}$ and $\left(\gamma+(1-\gamma) z^{-1}\right)^{-\alpha}$, it yields $(\alpha>0)$ :

$$
\begin{aligned}
\left(1-z^{-1}\right)^{\alpha} & =\sum_{k=0}^{\infty}(-1)^{k}\left(\begin{array}{l}
\alpha \\
k
\end{array}\right) z^{-k} \\
& =c_{0}^{(\alpha)}+c_{1}^{(\alpha)} z^{-1}+c_{2}^{(\alpha)} z^{-2}+\cdots+ \\
& =\sum_{k=0}^{\infty} c_{k}^{(\alpha)} z^{-k},
\end{aligned}
$$

$$
\begin{aligned}
\left(\gamma+(1-\gamma) z^{-1}\right)^{-\alpha} & =\sum_{k=0}^{\infty} \gamma^{-(\alpha+k)}(1-\gamma)^{k}\left(\begin{array}{c}
-\alpha \\
k
\end{array}\right) z^{-k} \\
& =d_{0}^{(-\alpha)}+d_{1}^{(-\alpha)} z^{-1}+\cdots+ \\
& =\sum_{k=0}^{\infty} d_{k}^{(-\alpha)} z^{-k}
\end{aligned}
$$


Let us introduce the product of the two generating series as:

$$
\begin{gathered}
\left(\sum_{k=0}^{\infty} c_{k}^{(\alpha)} z^{-k}\right)\left(\sum_{k=0}^{\infty} d_{k}^{(-\alpha)} z^{-k}\right) \\
=\sum_{k=0}^{\infty}\left(\sum_{j=0}^{k} c_{j}^{(\alpha)} d_{k-j}^{(-\alpha)}\right) z^{-k} .
\end{gathered}
$$

Using Taylor series (11) and (12) in conjunction with the product property (13), we obtain the final expansion of $H^{\alpha}\left(z^{-1}\right)$ into a power series in $z^{-1}$ :

$$
\begin{aligned}
H^{\alpha}\left(z^{-1}\right)= & \left(\frac{1}{\lambda T} \frac{1-z^{-1}}{\gamma+(1-\gamma) z^{-1}}\right)^{\alpha} \\
= & \left(\frac{1}{\lambda T}\right)^{\alpha} \sum_{k=0}^{\infty}\left(\sum_{j=0}^{k} \gamma^{-(\alpha+k-j)}(1-\gamma)^{k-j}\right. \\
& \left.\times\left(\begin{array}{c}
j-\alpha-1 \\
j
\end{array}\right)\left(\begin{array}{c}
-\alpha \\
k-j
\end{array}\right)\right) z^{-k} \\
= & h^{\alpha}(0)+h^{\alpha}(1) z^{-1}+h^{\alpha}(2) z^{-2}+\cdots+ \\
= & \sum_{k=0}^{\infty} h^{\alpha}(k) z^{-k},
\end{aligned}
$$

where the impulse response sequence $h^{\alpha}(k)$ is calculated by the expression $(k \geqslant 0)$ :

$$
\begin{aligned}
& h^{\alpha}(k)=\left(\frac{1}{\lambda T}\right)^{\alpha} \sum_{j=0}^{k} \gamma^{-(\alpha+k-j)}(1-\gamma)^{k-j} \\
& \times\left(\begin{array}{c}
j-\alpha-1 \\
:
\end{array}\right)\left(\begin{array}{c}
-\alpha \\
, .2
\end{array}\right) .
\end{aligned}
$$

Table 2 shows the impulse response sequences for the most commonly used discretization schemes, i.e., the Euler, the Tustin and the Al-Alaoui operators. These were obtained after simplification of expression (15) and considering the tuning parameter (with $\lambda=1) \gamma=1, \frac{1}{2}$ and $\frac{7}{8}$, respectively.

Table 2

Impulse responses sequences of Euler (first backward difference), Al-Alaoui and Tustin operators $(\lambda=1)$

\begin{tabular}{ll}
\hline Method & $h^{\alpha}(k), k \geqslant 0$ \\
\hline Euler $(\gamma=1)$ & $\left(\frac{1}{T}\right)^{\alpha}\left(\begin{array}{c}k-\alpha-1 \\
k\end{array}\right)$ \\
Al-Alaoui $\left(\gamma=\frac{7}{8}\right)$ & $\left(\frac{8}{7 T}\right)^{\alpha} \sum_{j=0}^{k}\left(\frac{1}{7}\right)^{k-j}\left(\begin{array}{c}j-\alpha-1 \\
j\end{array}\right)\left(\begin{array}{c}-\alpha \\
k-j\end{array}\right)$ \\
Tustin $\left(\gamma=\frac{1}{)}\right)$ & $\left(\frac{2}{T}\right)^{\alpha} \sum_{j=0}^{k}\left(\begin{array}{c}j-\alpha-1 \\
j\end{array}\right)\left(\begin{array}{c}-\alpha \\
k-j\end{array}\right)$ \\
\hline
\end{tabular}

Note that the power series method leads to impulse response sequences of infinite length. In a practically realizable form these sequences are truncated yielding approximations in the form of finite impulse responses (FIR filters).

\section{Design of IIR approximations to fractional} differintegrators using least-squares

Consider that the desired impulse response $h^{\alpha}(k)$ is specified for $k \geqslant 0$. The transfer function of the approximation $H\left(z^{-1}\right)$, to be designed, has the form

$H(z)=\frac{B(z)}{A(z)}=\frac{b_{0}+b_{1} z^{-1}+\cdots+b_{m} z^{-m}}{1+a_{1} z^{-1}+\cdots+a_{n} z^{-n}}$,

where $m \leqslant n$. The impulse response $h(k)$ is related to $H(z)$ by the $z$-transform

$H(z)=\sum_{k=0}^{\infty} h(k) z^{-k}$.

The approximation (16) has $m+n+1$ parameters, namely, the coefficients $a_{k}(k=1,2, \ldots, n)$ and $b_{k}$ $(k=0,1, \ldots, m)$, which can be selected to minimize the sum of the squared errors

$E=\sum_{k=0}^{N-1}\left[h^{\alpha}(k)-h(k)\right]^{2}$,

where $N$ denotes the number of impulse values used in the summation. In general, this approach leads to a nonlinear problem for the model parameters $\left(a_{k}\right.$, $b_{k}$ ) and hence the minimization of $E$ involves the solution of a set of nonlinear equations. However, if we rewrite $H(z)=B(z) / A(z)$ as follows:

$H(z) A(z)=B(z)$

and assuming that $h^{\alpha}(k)$ is given approximately by the impulse response of $H(z)$, one can write the corresponding time-domain equation as (note that the left-hand sided corresponds to a convolution)

$$
h^{\alpha}(k)+\sum_{l=1}^{n} a_{l} h^{\alpha}(k-l)= \begin{cases}b_{k}, & 0 \leqslant k \leqslant m, \\ 0, & k>m .\end{cases}
$$

This gives a set of linear equations, which can be used in different ways to solve for the coefficients $a_{k}$ and $b_{k}$ [19-24]. Our objective is to use simple (indirect) methods that can handle more easily the (indirect) methods that can handle more easily the determination of the model parameters. In this perspective, this study considers the application $\mathrm{O}$ solutions: the Pade'approximation, the Prony's method and the Shanks' method. 
The Padé fraction yields an approximation that fits exactly $h^{\alpha}(k)$ during the first $m+n+1$ values of $k$. Then, Eq. (20) becomes

$h^{\alpha}(k)+\sum_{l=1}^{n} a_{l} h^{\alpha}(k-l)= \begin{cases}b_{k}, & 0 \leqslant k \leqslant m, \\ 0, & m+1 \leqslant k \leqslant m+n,\end{cases}$

where $h^{z}(k)=0$ for $k<0$. A two-step approach is used to solve the system of $m+n+1$ linear equations in $m+n+1$ unknowns (21). In the first step, the coefficients $a_{k}$ are found using the last $n$ equations in (21), which after simple manipulations, may be written in matrix form as:

$$
\begin{gathered}
{\left[\begin{array}{cccc}
h^{\alpha}(m) & h^{\alpha}(m-1) & \cdots & h^{\alpha}(m-n+1) \\
h^{\alpha}(m+1) & h^{\alpha}(m) & \cdots & h^{\alpha}(m-n+2) \\
\vdots & \vdots & \ddots & \vdots \\
h^{\alpha}(m+n-1) & h^{\alpha}(m+n-2) & \cdots & h^{\alpha}(m)
\end{array}\right]} \\
\times\left[\begin{array}{c}
a_{1} \\
a_{2} \\
\vdots \\
a_{n}
\end{array}\right]=-\left[\begin{array}{c}
h^{\alpha}(m+1) \\
h^{\alpha}(m+2) \\
\vdots \\
h^{\alpha}(m+n)
\end{array}\right],
\end{gathered}
$$

$\mathbf{H}_{2} \mathbf{a}=-\mathbf{h}_{21}$,

where a and $\mathbf{h}_{21}$ are $n \times 1$ vectors and $\mathbf{H}_{2}$ is an $n \times n$ nonsymmetric Toeplitz matrix [25]. If $\mathbf{H}_{2}$ is nonsingular (i.e., is invertible) then $\mathbf{H}_{2}^{-1}$ exists and the coefficients $a_{k}(k=1,2, \ldots, n)$ may be uniquely determined by:

$\mathbf{a}=-\mathbf{H}_{2}^{-1} \mathbf{h}_{21}$.

In the second step, the coefficients $b_{k}$ are found using the first $m+1$ equations in (21), which may be written in matrix form as:

$$
\left[\begin{array}{ccccc}
h^{\alpha}(0) & 0 & 0 & \cdots & 0 \\
h^{\alpha}(1) & h^{\alpha}(0) & 0 & \cdots & 0 \\
h^{\alpha}(2) & h^{\alpha}(1) & h^{\alpha}(0) & \cdots & 0 \\
\vdots & \vdots & \vdots & \ddots & \vdots \\
h^{\alpha}(m) & h^{\alpha}(m-1) & h^{\alpha}(m-2) & \cdots & h^{\alpha}(m-n)
\end{array}\right]
$$

$$
\times\left[\begin{array}{c}
1 \\
a_{1} \\
a_{2} \\
\vdots \\
a_{n}
\end{array}\right]=\left[\begin{array}{c}
b_{0} \\
b_{1} \\
b_{2} \\
\vdots \\
b_{m}
\end{array}\right]
$$

$\mathbf{H}_{1} \mathbf{a}=\mathbf{b}$,

where $\mathbf{b}$ is an $(m+1) \times 1$ vector, $\mathbf{a}=[1 ; \mathbf{a}]$ is an $(n+1) \times 1$ vector and $\mathbf{H}_{1}$ is an $(m+1) \times(n+1)$ matrix. Therefore, $\mathbf{b}$ may be found simply by multiplying $\overline{\mathbf{a}}$ by $\mathbf{H}_{1}$. Alternatively, the coefficients $b_{k}$ may be evaluated using Eq. (21) as follows:

$b_{k}=h^{\alpha}(k)+\sum_{l=1}^{n} a_{l} h^{\alpha}(k-l), \quad k=0,1, \ldots, m$.

In this way, we obtain an approximation that has a perfect match to the desired impulse sequence $h^{\alpha}(k)$ for the first $m+n+1$ values of $k$. However, since there is no bound on the error for $k>m+n$, in general the Padé method does not produces a good approximation to $h^{\alpha}(k)$ for $k>m+n$. In fact, the success of this method depends strongly on the number of selected model coefficients. Since the design method matches $h^{\alpha}(k)$ only up to the number of model parameters, the more complex the model, the better is the approximation to $h^{\alpha}(k)$ for $0 \leqslant k \leqslant m+n$. However, in practical applications, this introduces a major limitation of the Padé method because the resulting approximation must contain a large number of poles and zeros [26].

It can be shown that rational approximations obtained by the CFE method are identical to those resulting by application of the Padé approximation to PSE $(m=n)$ [27]. Nevertheless, the CFE approach is computationally less expensive than the Padé technique.

\subsection{Prony's method}

Prony's method differs from the Padé approximation in the form of finding the coefficients $a_{k} \quad(k=1,2, \ldots, n)$. These are determined by a least-squares minimization over the interval 
$[m+1, N-1]^{1}:$

$E_{\mathrm{P}}=\sum_{k=m+1}^{N-1}\left|e_{\mathrm{P}}(k)\right|^{2}$,

where

$e_{\mathrm{P}}(k)=h^{z}(k)+\sum_{l=1}^{n} a_{l} h^{z}(k-l)$.

The coefficients $a_{k}$, that minimize $E_{\mathrm{P}}$, may be found by setting the error $e_{\mathrm{P}}(k)=0(k=m+1, m+$ $2, \ldots, N-1)$ and solving for the unknowns $a_{k}$. This produces the following set of linear equations

$$
\begin{gathered}
{\left[\begin{array}{cccc}
h^{\alpha}(m) & h^{\alpha}(m-1) & \cdots & h^{\alpha}(m-n+1) \\
h^{\alpha}(m+1) & h^{\alpha}(m) & \cdots & h^{\alpha}(m-n+2) \\
\vdots & \vdots & \ddots & \vdots \\
h^{\alpha}(N-2) & h^{\alpha}(N-3) & \cdots & h^{\alpha}(N-n-1)
\end{array}\right]} \\
\times\left[\begin{array}{c}
a_{1} \\
a_{2} \\
\vdots \\
a_{n}
\end{array}\right]=-\left[\begin{array}{c}
h^{\alpha}(m+1) \\
h^{\alpha}(m+2) \\
\vdots \\
h^{\alpha}(N-1)
\end{array}\right],
\end{gathered}
$$

$\mathbf{H}_{2} \mathbf{a}=-\mathbf{h}_{21}$,

where a is an $n \times 1$ vector, $\mathbf{h}_{21}$ is an $(N-m-1) \times 1$ vector and $\mathbf{H}_{2}$ is an $(N-m-1) \times n$ matrix. It is obvious that in this case (31) cannot be solved exactly. Therefore, the least-squares solution is obtained by solving the set of $n$ linear equations:

$\left(\mathbf{H}_{2}^{\mathrm{T}} \mathbf{H}_{2}\right) \mathbf{a}=-\mathbf{H}_{2}^{\mathrm{T}} \mathbf{h}_{21}$.

If the matrix $\mathbf{H}_{2}^{\mathrm{T}} \mathbf{H}_{2}$ is nonsingular, a unique solution of (32) exists and the coefficients $a_{k}$ are given by:

$\mathbf{a}=-\left(\mathbf{H}_{2}^{\mathrm{T}} \mathbf{H}_{2}\right)^{-1} \mathbf{H}_{2}^{\mathrm{T}} \mathbf{h}_{21}=-\mathbf{H}_{2}^{+} \mathbf{h}_{21}$,

where $\mathbf{H}_{2}^{+}=\left(\mathbf{H}_{2}^{\mathrm{T}} \mathbf{H}_{2}\right)^{-1} \mathbf{H}_{2}^{\mathrm{T}}$ is the pseudoinverse of $\mathrm{H}_{2}$.

Once the coefficients $a_{k}$ can be determined, the coefficients $b_{k}$ are found using the Padé method of forcing $h(k)=h^{\alpha}(k)$ for $k=0,1, \ldots, m$ (see the

\footnotetext{
${ }^{1}$ Note that this procedure corresponds to the Pade'method by relaxing the constraint given by Eq. (21) from $k m \mathbf{p} 1 ; m \mathbf{p} n]$ upon to the number of impulse samples under consideration, lim $\mathbf{p} 1 ; N-1]$.
}

$b_{k}=h^{\alpha}(k)+\sum_{l=1}^{n} a_{l} h^{\alpha}(k-l), \quad 0 \leqslant k \leqslant m$.

\subsection{Shanks' method}

Shanks' method provides an alternative to Prony's method of finding the coefficients $b_{k}$ $(k=0,1, \ldots, m)$. Instead of forcing an exact fit for the first $m+1$ values of the impulse response, it establishes a least squares minimization over the entire interval under consideration, $[0, N-1]^{2}$ :

$E_{\mathrm{S}}=\sum_{k=0}^{N-1}\left|e_{\mathrm{S}}(k)\right|^{2}$,

where

$e_{\mathrm{S}}(k)=h^{\alpha}(k)-\sum_{l=0}^{m} b_{l} g(k-l)$

In this approach, we convert the rational function $H(z)$ as the cascade of two functions:

$H(z)=B(z)\left\{\frac{1}{A(z)}\right\}$.

Firstly, the coefficients $a_{k}$ are determined in the same way as in Prony's method, i.e., by a least squares fit over the interval $[m+1, N-1]$ (see the previous subsection). Once $A(z)$ has been determined, the impulse response $g(k)$, which corresponds to the filter $1 / A(z)$, may be computed using, for example, the recursion

$$
\begin{aligned}
g(k) & =\delta(k)-\sum_{l=1}^{n} a_{l} g(k-l), \\
k & =0,1, \ldots, N-1
\end{aligned}
$$

with $g(k)=0$ for $k<0$.

Finally, the coefficients $b_{k}$, that minimize $E_{\mathrm{S}}$, may be found by setting the error $e_{\mathrm{S}}(k)=0$ $(k=0,1, \ldots, N-1)$, leading to the following set

\footnotetext{
${ }^{2}$ It is possible to make use of other errors. For instance, we may find it more appropriate to consider the same interval, l $m$ p $1 ; N-1]$, as that used in Prony's method to derive the coefficients $a_{k}$.
} 
Table 3

Coefficients of Prony's approximations, $H(z)=B(z) / A(z)$, to Tustin operator for $\alpha=-\frac{1}{2}, N=1000, T=0.01 \mathrm{~s}$ and $(m, n)=$ $\{(1,1),(3,3), \ldots,(9,9)\}$

\begin{tabular}{|c|c|c|c|c|c|c|}
\hline \multicolumn{2}{|c|}{ Rational function } & \multicolumn{5}{|l|}{ Order $(m, n)$} \\
\hline$H(z)$ & Coefficient & $(1,1)$ & $(3,3)$ & $(5,5)$ & $(7,7)$ & $(9,9)$ \\
\hline \multirow[t]{10}{*}{$B(z)$} & $b_{0}$ & 0.0707106781 & 0.0707106781 & 0.0707106781 & 0.0707106781 & 0.0707106781 \\
\hline & $b_{1}$ & 0.0088431239 & 0.0053020645 & 0.0047276912 & 0.0047370173 & 0.0049888640 \\
\hline & $b_{2}$ & & -0.0528109675 & -0.0939641699 & -0.1354562658 & -0.1770261981 \\
\hline & $b_{3}$ & & -0.0017470271 & -0.0043558157 & -0.0071653187 & -0.0104911076 \\
\hline & $b_{4}$ & & & 0.0265629907 & 0.0770957483 & 0.1521127174 \\
\hline & $b_{5}$ & & & 0.0005193119 & 0.0027898389 & 0.0070651570 \\
\hline & $b_{6}$ & & & & -0.0118029031 & -0.0506123212 \\
\hline & $b_{7}$ & & & & -0.0001729292 & -0.0015867209 \\
\hline & $b_{8}$ & & & & & 0.0049013950 \\
\hline & $b_{9}$ & & & & & 0.0000603757 \\
\hline \multirow[t]{10}{*}{$A(z)$} & $a_{0}$ & 1.0000000000 & 1.0000000000 & 1.0000000000 & 1.0000000000 & 1.0000000000 \\
\hline & $a_{1}$ & -0.8749393429 & -0.9250174848 & -0.9331403493 & -0.9330084584 & -0.9294468087 \\
\hline & $a_{2}$ & & -0.3218423797 & -0.8957136853 & -1.4826324234 & -2.0740816932 \\
\hline & $a_{3}$ & & 0.2596444279 & 0.8006833232 & 1.3478037438 & 1.8904384303 \\
\hline & $a_{4}$ & & & 0.1144011115 & 0.5753152257 & 1.3875245003 \\
\hline & $a_{5}$ & & & -0.0846141199 & -0.4935684346 & -1.2222439076 \\
\hline & $a_{6}$ & & & & -0.0415439761 & -0.3186806805 \\
\hline & $a_{7}$ & & & & 0.0278508899 & 0.2604191128 \\
\hline & $a_{8}$ & & & & & 0.0152884314 \\
\hline & $a_{9}$ & & & & & -0.0091873966 \\
\hline
\end{tabular}

Table 4

Coefficients of Prony's approximations, $H(z)=B(z) / A(z)$, to Al-Alaoui operator for $\alpha=-\frac{1}{2}, N=1000, T=0.01 \mathrm{~s}$ and $(m, n)=$ $\{(1,1),(3,3), \ldots,(9,9)\}$

\begin{tabular}{|c|c|c|c|c|c|c|}
\hline \multicolumn{2}{|c|}{ Rational function } & \multicolumn{5}{|l|}{ Order $(m, n)$} \\
\hline$H(z)$ & Cocfficient & $(1,1)$ & $(3,3)$ & $(5,5)$ & $(7,7)$ & $(9,9)$ \\
\hline \multirow[t]{10}{*}{$B(z)$} & $b_{0}$ & 0.0935414347 & 0.0935414347 & 0.0935414347 & 0.0935414347 & 0.0935414347 \\
\hline & $b_{1}$ & -0.0336832200 & -0.1310909495 & -0.2293618467 & -0.3276101609 & -0.4257543935 \\
\hline & $b_{2}$ & & 0.0385810145 & 0.1860475050 & 0.4366839696 & 0.7901053063 \\
\hline & $b_{3}$ & & 0.0020712409 & -0.0506646281 & -0.2684862300 & -0.7591515747 \\
\hline & $b_{4}$ & & & -0.0002192801 & 0.0687844321 & 0.3906490449 \\
\hline & $b_{5}$ & & & 0.0007631060 & -0.0015285715 & -0.0948889202 \\
\hline & $b_{6}$ & & & & -0.0014280565 & 0.0034735963 \\
\hline & $b_{7}$ & & & & 0.0000464547 & 0.0022157823 \\
\hline & $b_{8}$ & & & & & -0.0001824620 \\
\hline & $b_{9}$ & & & & & -0.0000077165 \\
\hline \multirow[t]{10}{*}{$A(z)$} & $a_{0}$ & 1.0000000000 & 1.0000000000 & 1.0000000000 & 1.0000000000 & 1.0000000000 \\
\hline & $a_{1}$ & -0.9315173402 & -1.9728497700 & -3.0234098516 & -4.0737285101 & -5.1229344900 \\
\hline & $a_{2}$ & & 1.1316278541 & 3.3084309965 & 6.5880295990 & 10.9658088885 \\
\hline & $a_{3}$ & & -0.1574496076 & -1.5363072377 & -5.3102729619 & -12.6290469641 \\
\hline & $a_{4}$ & & & 0.2527828079 & 2.1636134979 & 8.3546323360 \\
\hline & $a_{5}$ & & & -0.0014666745 & -0.3768952461 & -3.0965746303 \\
\hline & $a_{6}$ & & & & 0.0067998050 & 0.5485273792 \\
\hline & $a_{7}$ & & & & 0.0024545385 & -0.0146333039 \\
\hline & $a_{8}$ & & & & & -0.0060623532 \\
\hline & $a_{9}$ & & & & & 0.0002831560 \\
\hline
\end{tabular}


of linear equations

$$
\begin{gathered}
{\left[\begin{array}{cccc}
g(0) & 0 & 0 & 0 \\
g(1) & g(0) & 0 & 0 \\
g(2) & g(1) & 0 & 0 \\
\vdots & \vdots & \ddots & \vdots \\
g(N-1) & g(N-2) & \cdots & g(N-m-1)
\end{array}\right]} \\
\times\left[\begin{array}{c}
b_{0} \\
b_{1} \\
\vdots \\
b_{m}
\end{array}\right]=\left[\begin{array}{c}
h^{\alpha}(0) \\
h^{\alpha}(1) \\
h^{\alpha}(2) \\
\vdots \\
h^{\alpha}(N-1)
\end{array}\right],
\end{gathered}
$$

$\mathbf{G b}=\mathbf{h}$,

where $\mathbf{b}$ is an $(m+1) \times 1$ vector, h is an $N \times 1$ vector and $\mathbf{G}$ is an $N \times(m+1)$ matrix. The leastsquares solution is found by solving a system of $m+1$ linear equations:

$\left(\mathbf{G}^{\mathrm{T}} \mathbf{G}\right) \mathbf{b}=\mathbf{G}^{\mathrm{T}} \mathbf{h}$.

If the matrix $\mathbf{G}^{\mathrm{T}} \mathbf{G}$ is nonsingular, the coefficients $a_{k}$ can be uniquely determined by:

$\mathbf{b}=\left(\mathbf{G}^{\mathrm{T}} \mathbf{G}\right)^{-1} \mathbf{G}^{\mathrm{T}} \mathbf{h}=\mathbf{G}^{+} \mathbf{h}$,

where $\mathbf{G}^{+}=\left(\mathbf{G}^{\mathrm{T}} \mathbf{G}\right)^{-1} \mathbf{G}^{\mathrm{T}}$ is the pseudoinverse of $\mathbf{G}$.

The techniques just described may be complemented with the following observations:

- The Prony and the Shanks methods are superior to Padé approximation, since $h(k)$ approximates $h^{\alpha}(k)$, in a least-squares sense, for values of $k>m+n$. Therefore, it will be expected a good match even outside the interval $[0, m+n]$;

- In the Shanks' method, both $a_{k}$ and $b_{k}$ are determined through an optimization viewpoint (using least-squares minimization), leading to a further improvement of the approximation accuracy. However, note that these optimization algorithms are distinct from the one obtained by the direct application of the least-squares method;

- The use of inverses or pseudoinverses is acceptable in theory, but practical calculations should better avoid them and solve linear algebraic systems, possibly in a least-squares sense. It is well known that there are efficient methods for the solution of such systems, either by using the linear equations (23), (32) and (41) [28], or using the inverses or pseudoinverses (24), (33) and (42) [29], but the best choice is probably the use of one of the least-squares solvers available. In this perspective, we verify that the use of the MATLAB backlash operator " " can easily and efficiently solve the algorithms proposed in this study.

\section{Illustrative examples}

Here we use the signal modeling techniques described in previous section to develop digital rational approximations $H\left(z^{-1}\right)$ of the continuous fractional-order operator $s^{z}, \alpha= \pm 1 / 2$, sampled at $T=0.01 \mathrm{~s}$. It is adopted an impulse sequence length

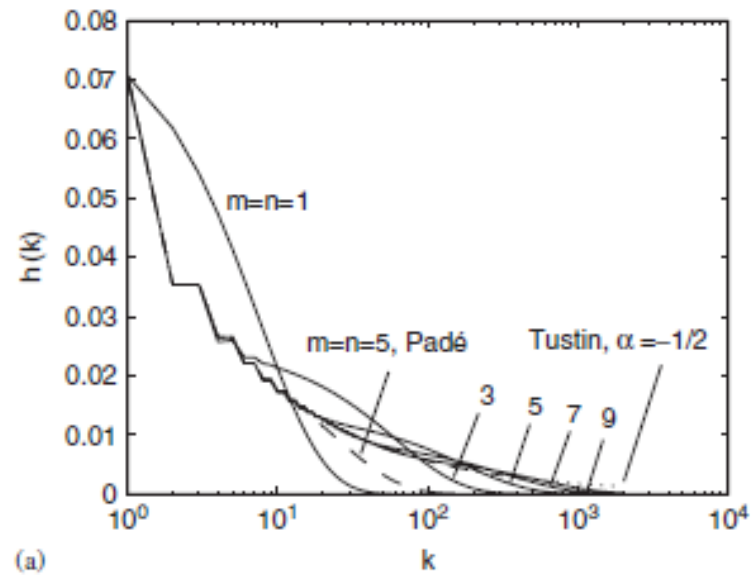

(a)

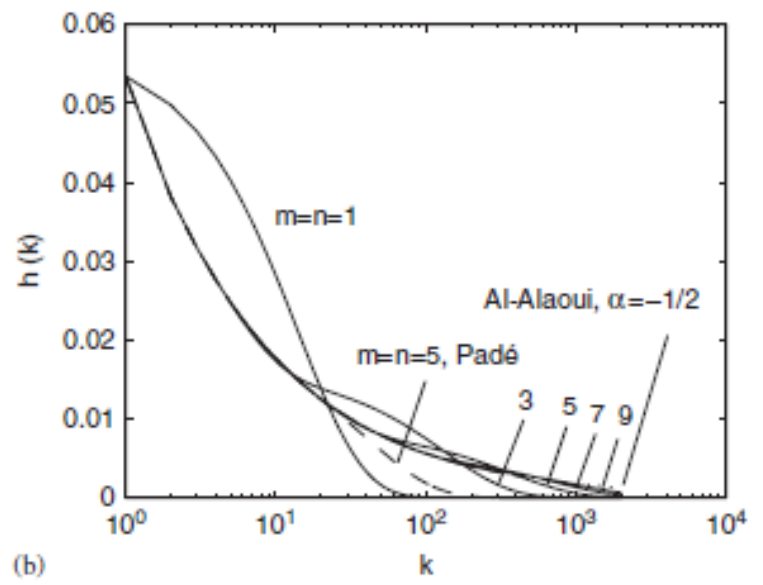

Fig. 1. Impulse response sequences of Prony's approximations to Tustin (upper) and Al-Alaoui (down) operators for $\alpha=-\frac{1}{2}$, $N=1000, T=0.01 \mathrm{~s}$ and $m=n=1,3, \ldots, 9$. 
of $N_{1 / 4}^{1} 1000$. In practice, we consider $m 1 / 4 n$ because the case of $m o n$ leads to inferior results $[11,19$, 24]. For comparison purposes, we also plot the rational approximation obtained by the Pade' (or the CFE) method for the case of $m / 4 \quad n k 5 / 4$. Tables 3 and 4 list the coefficients of selected Prony's approximations to Tustin and Al-Alaoui operators for a $1 / 4-\frac{1}{2}$ and $m^{1 / 4} n^{1 / 4} 1 ; 3 ; \ldots ; 9$, respectively.
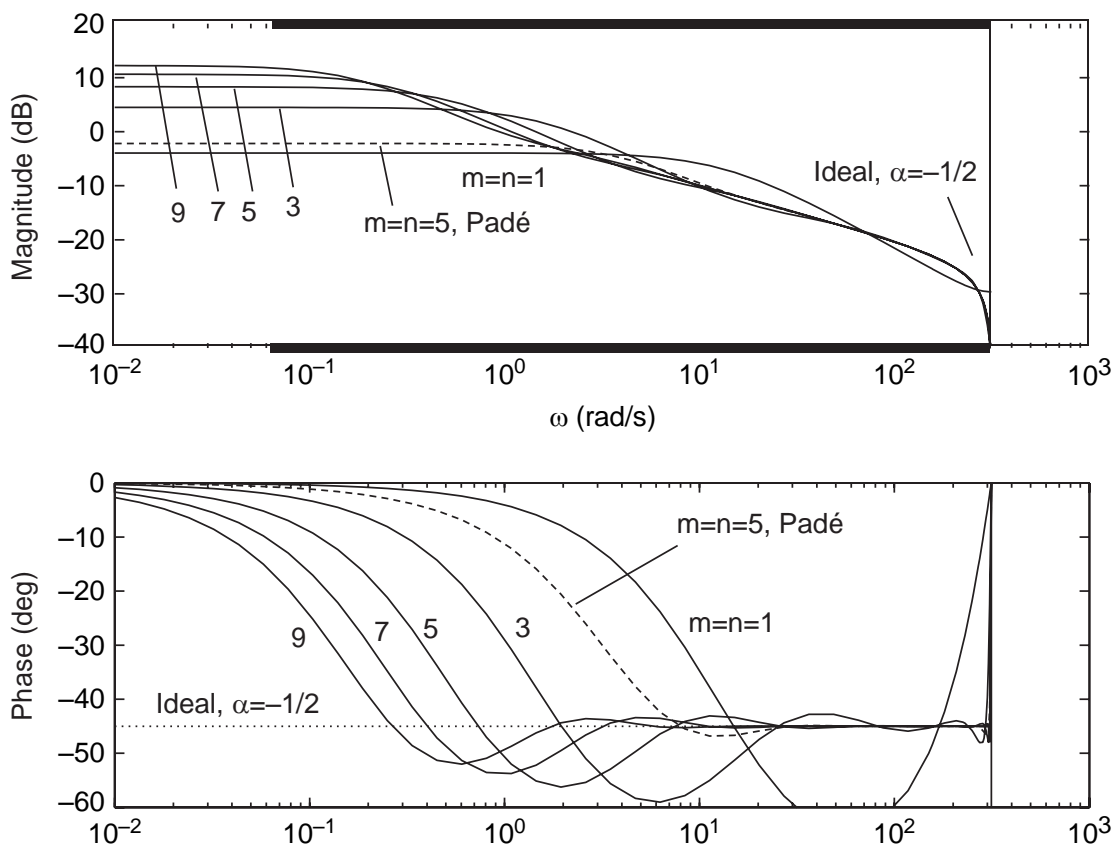

(a)

$\omega(\mathrm{rad} / \mathrm{s})$
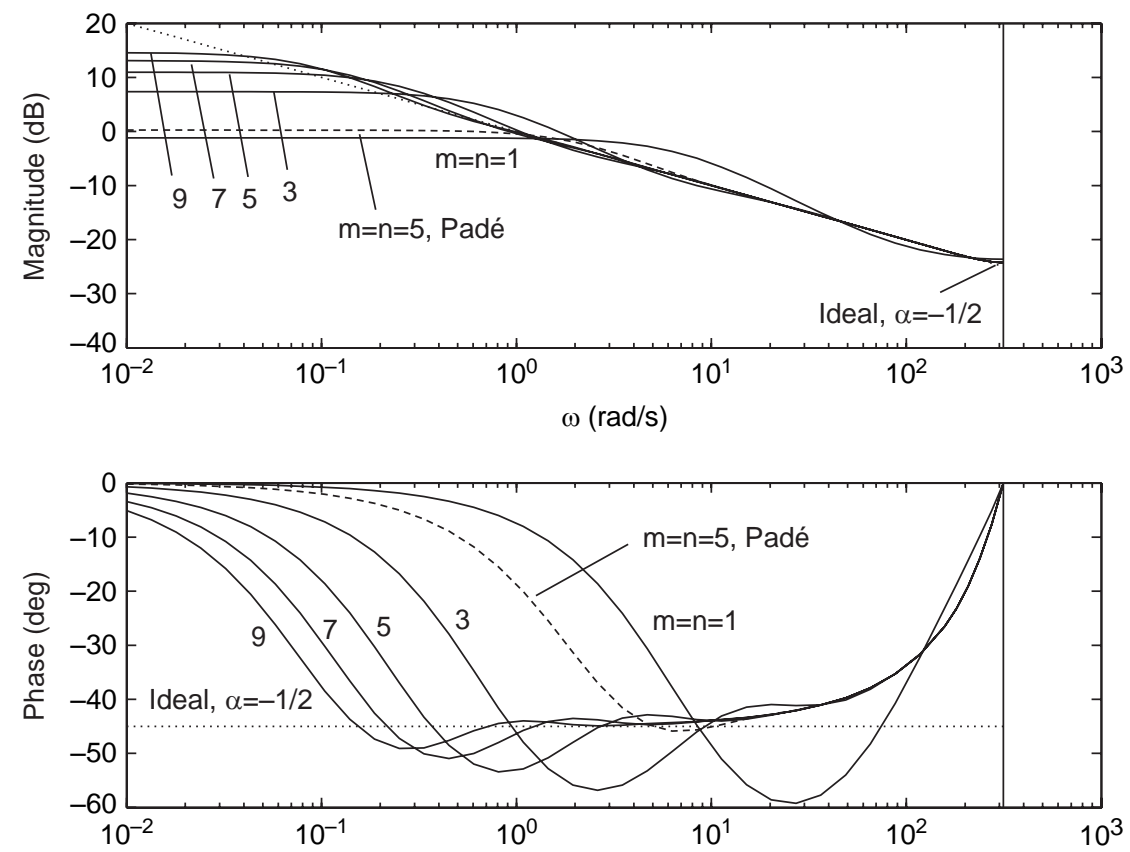

(b)

$\omega(\mathrm{rad} / \mathrm{s})$

Fig. 2. Bode diagrams of Prony's approximations to Tustin (upper) and Al-Alaoui (down) operators for $a_{2}^{1 / 4}-{ }^{1}, N^{1 / 4} 1000, T^{1 / 4} 0: 01 \mathrm{~s}$ and $m \frac{1}{4} n \frac{1}{4} 1 ; 3 ; . . . ; 9$. 

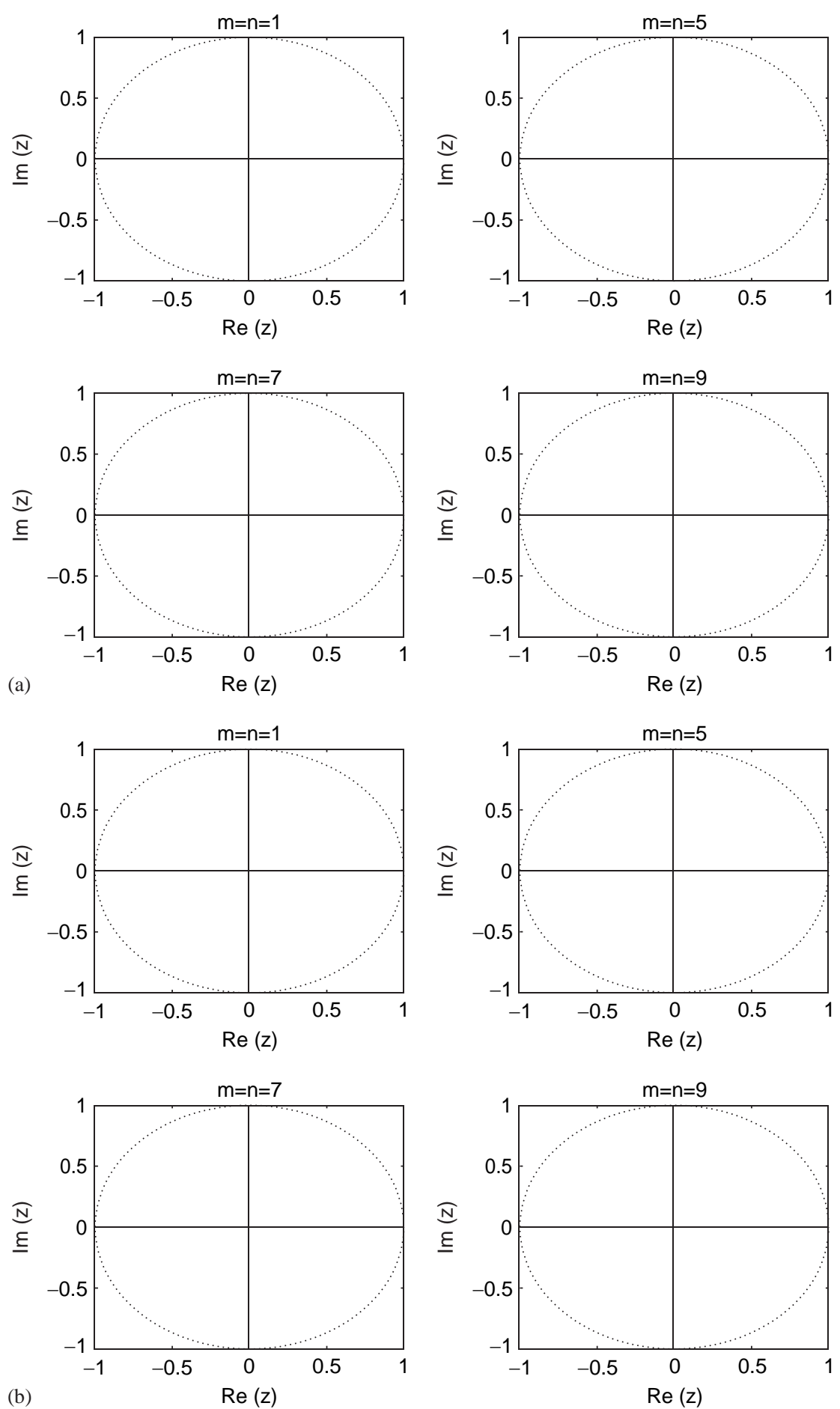

Fig. 3. Pole-zero maps of Prony's approximations to Tustin (upper) and Al-Alaoui (down) operators for $a_{2}{ }^{1 / 4}-{ }^{1}, N{ }^{1 / 4} 1000, T 1 / 40: 01 \mathrm{~s}$ and $m^{1 / 4} n^{1 / 4} \mathrm{fl} 15 ; 7 ; 9 \mathrm{~g}$. 


\subsection{Performance of the least-squares IIR approximations}

Figs. 1 and 2 depict the impulse response sequences and the Bode diagrams of Prony's approximations to Tustin and Al-Alaoui operators for a $1 / 4-{ }^{1}$ and $m^{1 / 4} n^{1 / 4} 1 ; 3 ; \ldots ; 9$, respectively.

Clearly, the higher the order $m \quad 1 / 4 n$ of the rational function better the fitting, in a least-squares sense, of its impulse response in the discretized fractionalorder integrator $s^{-1=2}$. Also, the Bode plots show that the approximations are well fitted into the ideal responses (dashed-dotted lines), roughly approximating the ideal continuous magnitude responses for nearly three decades (for $m^{1 / 4} n \times 5$ ). Note that the Al-Alaoui scheme improves the high frequency magnitude response comparatively to the Tustin scheme while this one has a better phase response approximation. We also verify that the least-squares approach increases the performance of the approximations in the low frequency range (corresponding to the steady-state time response) by increasing the order (or the number of impulse values used), resulting in better approximations than those given by the Pade' (or the CFE) method. This is due to the fact that the proposed techniques (Prony and Shanks) perform a least-squares fitting over a wide range of impulse samples, while the Pade'method produces an exact fit for the first $n \mathbf{p} 1$ samples of the impulse response, with any guarantee about the accuracy of the approximation for $k 4 m \mathrm{p} n$. Obviously, the upper limit frequency is dependent on the sampling period $T$ through the Nyquist criterion.

Fig. 3 shows the pole-zero maps of Prony's approximations to the Tustin and Al-Alaoui operators for a $1 / 4-\frac{1}{2}$ and $\left.m \quad 1 / 4 n \quad 1 / 4,5,7,9\right\}$. We observe that the approximations satisfy two desired properties: (i) all the poles and zeros lie inside the unit circle, and (ii) the poles and zeros are interlaced along the segment of the real axis corresponding to $z 21-1$. pThus, the resulting approximations are causal, stable and minimum phase, as desired for a digital realization.

\subsection{Fractional differintegration of some standard time-domain functions}

To further illustrate the effectiveness of the proposed techniques, the approximations are used to calculate the differintegral of the unit step function that occurs at $t 1 / 4 t_{0}\left(t_{0} 40\right), u \partial t-t_{0} \mathrm{P}$, and of the causal cosine function $c \succsim t \mathrm{p}$ defined as:

$$
\begin{aligned}
& u\left(t-t_{0}\right)= \begin{cases}1, & t \geqslant t_{0}, \\
0, & t<t_{0},\end{cases} \\
& c(t)=\cos (t) u(t) .
\end{aligned}
$$

The differintegral of the unit step function $u\left(t-t_{0}\right)$ is given by $[1,3]$

$$
D^{\alpha}\left[u\left(t-t_{0}\right)\right]= \begin{cases}\frac{\left(t-t_{0}\right)^{-\alpha}}{\Gamma(1-\alpha)}, & t>t_{0}, \\ 0, & 0 \leqslant t \leqslant t_{0} .\end{cases}
$$

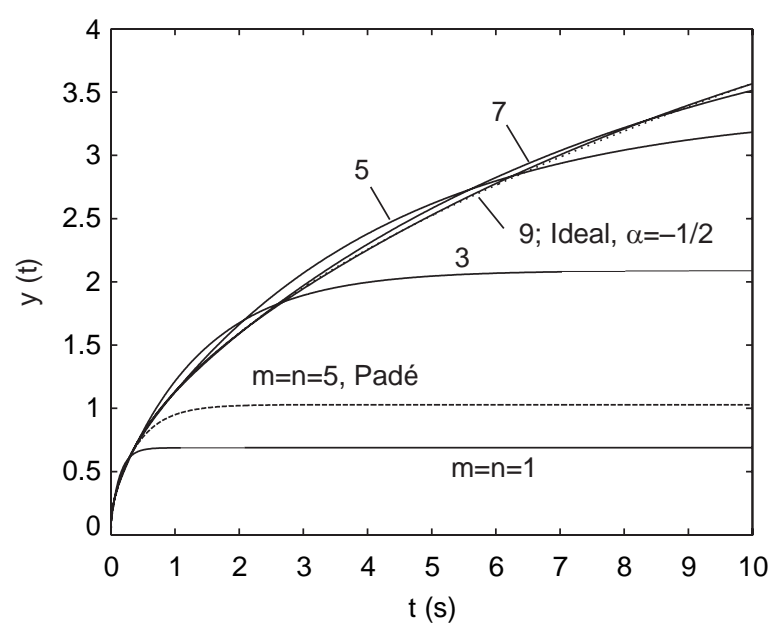

Fig. 4. Unit step responses of Shanks' approximations to AlAlaoui operator for a $1 / 4-\frac{1}{2}, \quad N 1 / 41000, \quad T 1 / 40: 01 \mathrm{~s}$ and $m^{1 / 4} n^{1 / 4} 1 ; 3 ; . . . ; 9$.

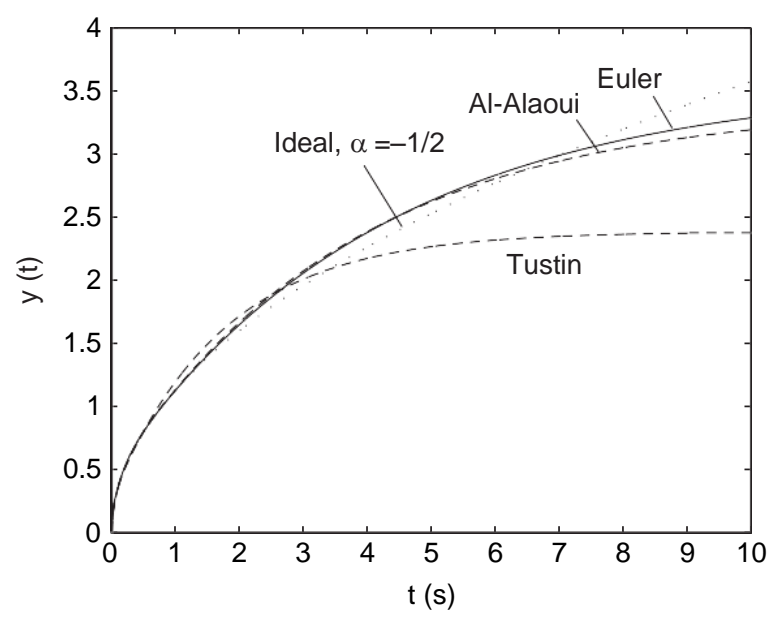

Fig. 5. Unit step responses of Shanks' approximations to Euler, Al-Alaoui and Tustin operators for a $1 / 4-\frac{1}{2}, N 1 / 41000, T 1 / 4$ 0:01 s and $m^{1 / 4} n^{1 / 4} 7$. 
The cosine function $c(t)$ is calculated for the semiderivative $\left(\alpha=\frac{1}{2}\right)$ and semiintegral $\left(\alpha=-\frac{1}{2}\right)[1]$ :

$D^{1 / 2}[\cos (t)]$

$$
=\frac{1}{\sqrt{\pi t}}+\cos \left(t+\frac{\pi}{4}\right)-\sqrt{2} f\left(\sqrt{\frac{2 t}{\pi}}\right),
$$

$$
D^{-1 / 2}[\cos (t)]=\cos \left(t-\frac{\pi}{4}\right)-\sqrt{2} g\left(\sqrt{\frac{2 t}{\pi}}\right),
$$

where $f(x)$ and $g(x)$ are the auxiliary Fresnel integrals [30]. In expressions (46) and (47), the cosine terms express the steady-state responses while the remaining terms represent the transient behaviour of the responses. In fact, considering $t \rightarrow \infty$, Eqs. (46), (47) are given by the general expression $D^{\alpha}[\cos (t)]=\cos (t+\alpha \pi / 2),-1<\alpha<1$.

Fig. 4 illustrates the unit step responses of Shanks' approximations to Al-Alaoui operator for several values of order $m n^{1 / 4} 1 ; 1 / 4 ; \ldots ; 9$. Once more, the curves show the good performance of the least-squares approximations in the time-domain comparatively to the Pade' (or the CFE) approximation. In Fig. 5 we compare the unit step responses of
Shanks' approximations with the three operators under consideration (Euler, Al-Alaoui and Tustin) for a fixed order of $m n$ 7. It is clear that the best approximations are obtained with the Euler and Al-Alaoui operators (note that the<smiles>CCCCCC[AlH]</smiles>

Al-Alaoui operator is a weighted interpolation of the Euler $ð-\mathrm{P}$ and the Tustin $ð \mathrm{P}$ operators). By other hand, as already said in previous subsection, the Tustin has the best frequency response. From these results, we conclude that the operators must be carefully selected depending on the final utilization of the approximations since they present different performances in the time and frequency domains that should be considered.

Figs. 6 and 7 illustrate the semiintegral (a $1 / 4-\frac{1}{2}$ ) and semiderivative (a $1 / 4 \frac{1}{z}$ ) of the functions $u t \partial-1 \mathrm{p}$ and $c$ ðtacalculated with the Shanks' and the Prony's approximations, respectively. The results demonstrate the effectiveness of the approximations fitting the ideal curves (dashed-dotted lines). Obviously, we can tune the order $m / 4 n$ of the approximation along with the sampling period $T$ to get a better agreement between the ideal and the approximated curves.
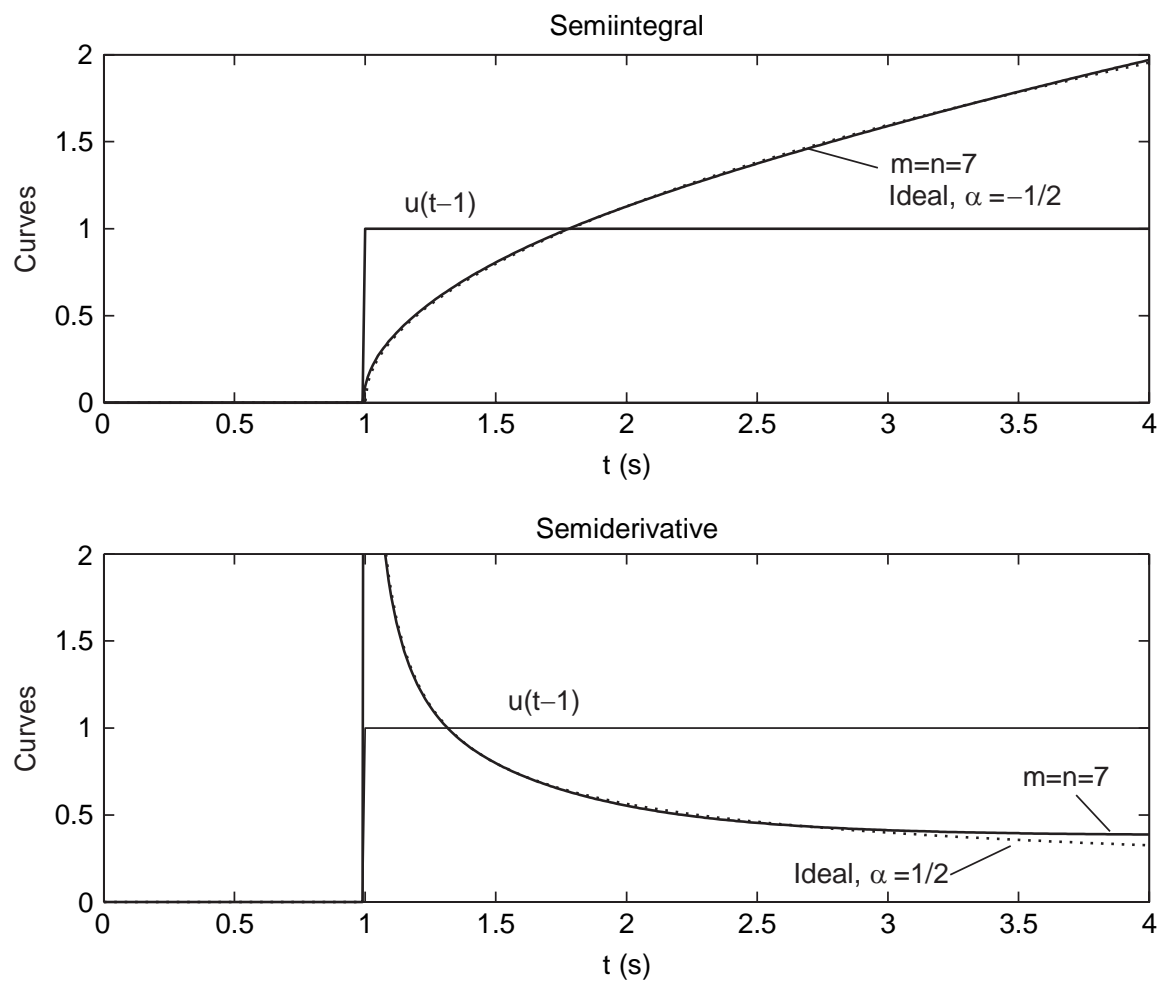

Fig. 6. Semiintegral and semiderivative of the unit step function $u ð t-1 \mathrm{P}$ calculated with Shanks' approximation to Al-Alaoui operator for $N^{1 / 4} 1000, T 1 / 40: 01 \mathrm{~s}$ and $m^{1 / 4} n^{1 / 4} 7$ : 

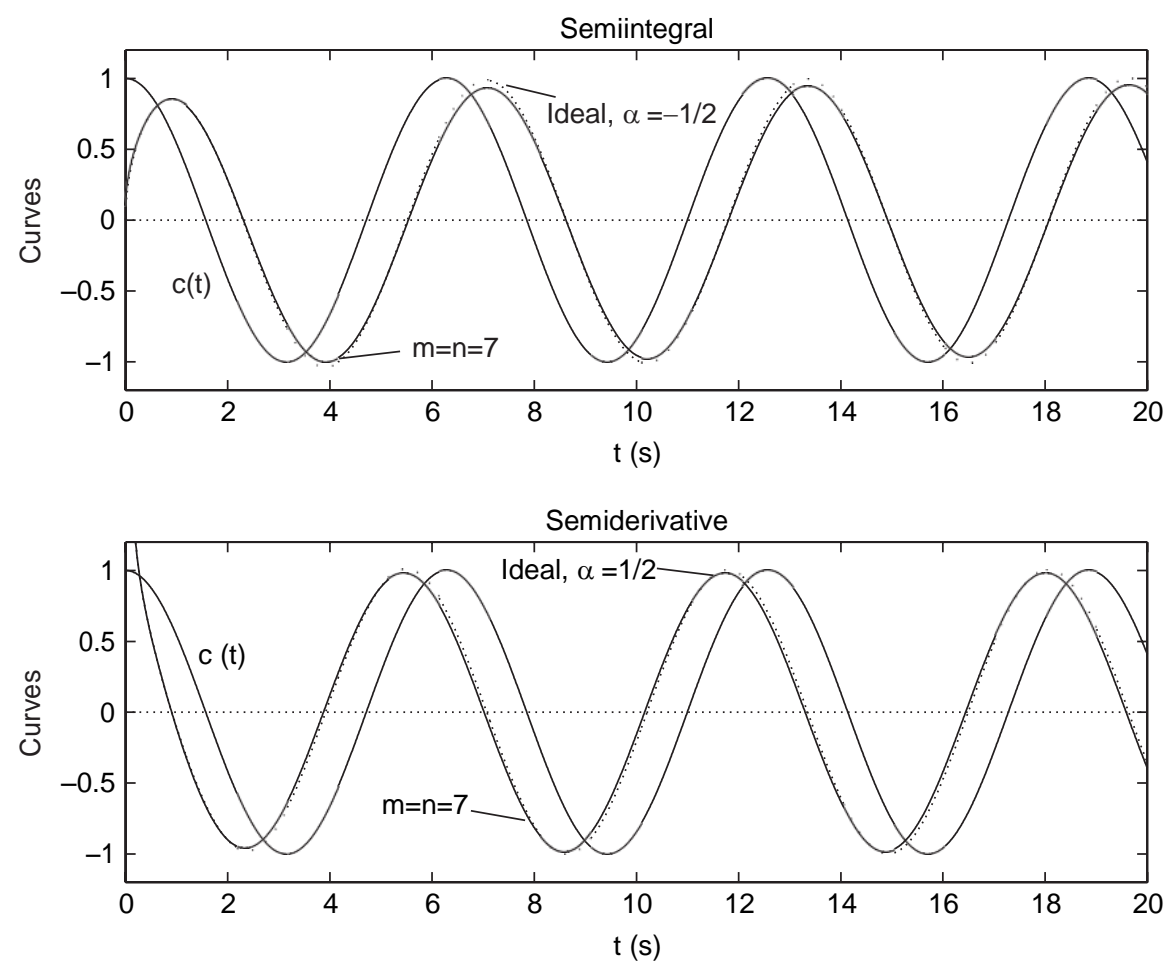

Fig. 7. Semiintegral and semiderivative of the cosine function $c \partial t$ calculated with Prony's approximation to Euler operator for $N 1 / 41000$, $T^{1 / 4}$ 0:01 s and $m^{1 / 4} n^{1 / 4} 7$ :

\section{Conclusions}

We have described the application of the Pade', Prony and Shanks techniques used for the signal modeling of deterministic signals to the design of digital rational approximations (IIR filters) of continuous fractional-order integrators and differentiators of type $s^{\mathrm{a}}$, $\mathrm{a} 2 \mathrm{R}$. It is shown that these techniques only require finding the solution to a set of linear equations. Note, however, that the illustrated techniques yield suboptimal solutions to the signal modeling problem, which differ from the optimal solution given by the direct application of the least squares method between the desired and the approximated impulse responses. This method has the disadvantage of requiring the solution of a set of nonlinear equations and, for that reason, it is often avoided.

The effectiveness of the approximations are illustrated both in the time and frequency domains. Moreover, it is demonstrated that the Prony and Shanks methods can produce better approximations than the widely used CFE approximation method. This is due to the fact that the poles (for the case of the Prony's method) and the zeros and poles (for the case of the Shanks' method) of the approximation are determined in a least squares sense over the (almost) entire impulse sequence length under consideration. By other hand, the Pade'approximation fits only on the desired impulse response, up to the number of poles and zeros, without any error control for larger values. In this case, can be easily proved that the Pade'and the CFE methods yield the same approximation $\left(\mathrm{ml}^{\mathrm{l}} / \mathrm{m}\right)$. Also, the obtained approximations are causal, stable and minimum phase, suitable for a real-time implementation.

The results presented here indicate that the leastsquares based methods are adequate techniques for obtaining digital approximations of continuous fractional-order operators. They also suggest the adoption of other similar procedures like the use of iterative methods (i.e., steep descent, Newton's method or iterative prefiltering). Although, these techniques are more involved than the methods presented here, they may produce interesting results (note that this is a batch process determination and the computation time is not a crucial issue). In this line of thought, this paper represents a step towards the implementation of practical digital fractionalorder differentiators and integrators. 


\section{References}

[1] K.B. Oldham, J. Spanier, The Fractional Calculus, Academic Press, New York, 1974.

[2] K.S. Miller, B. Ross, An Introduction to the Fractional Calculus and Fractional Differential Equations, Wiley, New York, 1993.

[3] I. Podlubny, Fractional Differential Equations, Academic Press, San Diego, 1999.

[4] A. Oustaloup, La Commande CRONE: Commande Robuste d'Ordre Non Entier, Editions Herme`s, Paris, 1991.

[5] A. Oustaloup, La Dérivation Non Entie` re: The'orie, Synthe`se et Applications, Editions Herme`s, Paris, 1995.

[6] R. Hilfer, Applications of Fractional Calculus in Physics, World Scientific, Singapore, 2000.

[7] I. Podlubny, Fractional-order systems and $\mathrm{PI}^{1} \mathrm{D}^{\mathrm{m}}$-controllers, IEEE Trans. Automat. Control 44 (1) (1999) $208-214$

[8] J.A.T. Machado, Analysis and design of fractional-order digital control systems, J. Syst. Anal., Model. Simul. 27 (1997) 107-122.

[9] J.A.T. Machado, Discrete-time fractional-order controllers, Fract. Calculus Appl. Anal. 4 (1) (2001) 47-66.

[10] B.M. Vinagre, I. Podlubny, A. Herna ndez, V. Feliu, Some approximations of fractional order operators used in control theory and applications, Fract. Calculus Appl. Anal. 3 (3) (2000) 231-248.

[11] Y.Q. Chen, K.L. Moore, Discretization schemes for fractional-order differentiators and integrators, IEEE Trans. Circuits and Systems-I: Fundam. Theory Appl. 49 (3) (2002) 363-367.

[12] M.A. Al-Alaoui, Novel digital integrator and differentiator, Electron. Lett. 29 (4) (1993) 376-378.

[13] M.A. Al-Alaoui, Filling the gap between the bilinear and the backward-difference transforms: an interactive design approach, Internat. J. Elec. Eng. Edu. 34 (4) (1997) 331337.

[14] J.M. Smith, Mathematical Modeling and Digital Simulation for Engineers and Scientists, second ed., Wiley, New York, 1987.

[15] C.C. Tseng, Design of fractional order digital fir differentiators, IEEE Signal Process. Lett. 8 (3) (2001) 77-79.

[16] B.M. Vinagre, Y.Q. Chen, I. Petras, Two direct tustin discretization methods for fractional-order differentiator/ integrator, J. Franklin Inst. 340 (2003) 349-362.
[17] Y.Q. Chen, B.M. Vinagre, A new IIR-type digital fractional order differentiator, Signal Processing 83 (11) (2003) 23592365.

[18] Y.Q. Chen, B.M. Vinagre, I. Podlubny, Continued fraction expansion approaches to discretizing fractional order derivatives-an expository review, Nonlinear Dyn. 38 (2004) $155-170$.

[19] R.S. Barbosa, J.A.T. Machado, I.M. Ferreira, Least-squares design of digital fractional-order operators, in: Proceedings of the First IFAC Workshop on Fractional Differentiation and its Applications (FDA'04), Bordeaux, France, July 1921, 2004, pp.434-439.

[20] T.H. Parks, C.S. Burrus, Digital Filter Design, Wiley, New York, 1987.

[21] M.H. Hayes, Statistical Digital Signal Processing and Modeling, Wiley, New York, 1996.

[22] Y. Ferdi, B. Boucheham, Recursive filter approximation of digital differentiator and integrator based on Prony's method, in: Proceedings of the First IFAC Workshop on Fractional Differentiation and its Applications (FDA'04), Bordeaux, France, July 19-21, 2004, pp. 428-433.

[23] M.D. Ortigueira, A.J. Serralheiro, New insights into pseudofractional ARMA modelling, in: Proceedings of the Second IEEE International Conference on Computational Cybernetics (ICCC 2004), Vienna, Austria, August 30-September 1, 2004, pp. 391-394.

[24] R.S. Barbosa, J.A.T. Machado, I.M. Ferreira, Pole-zero approximations of digital fractional-order integrators and differentiators using signal modeling techniques, in: 16th IFAC World Congress, Prague, Czech Republic, July 4-8, 2005.

[25] A.D. Poularikas, The Handbook of Formulas and Tables for Signal Processing, CRC Press, Boca Raton, FL, 1999.

[26] J.G. Proakis, D.G. Manolakis, Digital Signal Processing: Principles, Algorithms and Applications, third ed., PrenticeHall, Upper Saddle River, 1996.

[27] L. Lorentzen, H. Waadeland, Continued Fractions with Applications, Addison-Wesley, North-Holland, Amsterdam, 1992.

[28] R.L. Burden, J.D. Faires, Numerical Analysis, seventh ed., Brooks/Cole, Pacific Grove, CA, 2001.

[29] G.H. Golub, C.F.V. Loan, Matrix Computations, third ed., The Johns Hopkins University Press, Baltimore and London, 1996.

[30] M. Abramowitz, I.A. Stegun, Handbook of Mathematical Functions, Dover Publications, New York, 1974. 